Opinion

\title{
Probiotics food supplement for NAFLD
}

Keywords: triglycerides, lipopolysaccharides, adipose factor, lipogensis, pro biotics, food supplements

Abbreviations: NAFLD, non-alcoholic fatty liver disease; LPS, lipopolysaccharides; FIAF, fasting induced adipose factor; GIT, gastrointestinal tract; FAS, fatty acid synthesis gene

\section{Opinion}

Non-alcoholic fatty liver disease (NAFLD) is an accumulation of fat in triglycerides form in liver cells, it correlates well with obesity and it becomes a world health concern. Diet plays an important role in either increase or decreases the risk of NAFLD; increase consumption of fast foods, diet rich fats or fructose syrup correlates well with obesity and NAFLD. However, increase consumption of food rich in fiber results in decreasing the risk of NAFLD. Over growth of gut micro biota plays, also, an important role in developing of NAFLD through increase levels of production of endogenous ethanol, acetate, endotoxin lipopolysaccharides (LPS) which linked with increase the inflammation process, suppression of fasting induced adipose factor (FIAF) and increase lipogensis.

Probiotics are live microbial strains with health impact on host when they consumed daily with enough amounts (not less than $10^{6}$ to $10^{8} \mathrm{CFU} / \mathrm{g}$ ) and incorporated into gut micro biome. Different food products could be a carrier for pro biotics e.g. fermented milks, cheeses, ice-cream, beverages and fermented meat products. For enhancing the viability of pro biotics in different products or during gastrointestinal tract (GIT) conditions, microencapsulation technologies provide a useful strategy for protecting different probiotic strains from different harsh conditions especially those in GIT. Different probiotic strains (can decrease the risk of NAFLD through different mechanisms including:

i. Modulation of gut microbiota composition towards healthy directions.

ii. Modulation of gut microbiota metabolites.

iii. Modulation of lipid metabolism including reduction of levels of plasma cholesterol.

iv. Modulation of different gene expression relates to lipogensis e.g. fatty acid synthesis gene (FAS).

\author{
Volume 6 Issue 4 - 2017
}

\section{Fouad M F Elshaghabee}

Department of Dairy Science, Cairo University, Egypt

Correspondence: Fouad MF Elshaghabee, Department of Dairy Science, Faculty of Agriculture, Cairo University, $3 \mathrm{El}$ Gamaa St, 12613 Giza, Egypt,

Email elshaghabee@daad-alumni.de

Received: May 12, 2017 | Published: May 18, 2017

v. Enhancement the intestinal tight junction.

vi. Decrease the inflammatory process that associates with NAFLD.

In pharma-shops, VSL\#3 probiotic mixture had approved a good probiotic complementary therapy for reducing the risk of NAFLD. However, in my opinion, these results differ between different groups of patients due to, for example, differences in life style, geographic factor, age, and genes variance. Also, there is still gap of knowledge to understand by which mechanism of action of different probiotic food supplements could decrease the risk of NAFLD. Finally, we should increase the awareness of consumer with healthy foods and daily dose of pro biotics and teach people what they should eat for enhancing the public health.

\section{Acknowledgements}

None.

\section{Conflict of interest}

Author declares that there is no conflict of interest. 